\title{
The Cost Reduction Research of GPTMS-Boehmite Transparent and Hard Coating Film
}

\author{
Kui $\mathrm{CHEN}^{1, \text { a }}$, Tian-Yun ZHANG ${ }^{2, \text { b }}$, Xiao-Ping ZHENG ${ }^{1, c}$ \\ ${ }^{1}$ School of Beilie Engineering \& Technology, Lanzhou City University, Lanzhou 730070, China \\ ${ }^{2}$ Center of Information and Network, Lanzhou City University, Lanzhou 730070, China \\ achenkui405@126.com, ${ }^{\mathrm{b}}$ Izyunyun@126.com, ${ }^{\mathrm{c}} \mathrm{zxp} @$ Izcu.edu.cn
}

Keywords: Organo Alkoxy Silane, Aluminium Isopropoxide, Film, Transparency.

\begin{abstract}
In order to reduce cost and contain properties, GPTMS-boehmite transparent and hard coating film was prepared by replacing boehmite with aluminium isopropoxide (AIP), and modified by hydrolyzed TEOS. By the research of the process, a cheap transparent coating film with a pencil hardness of $4 \mathrm{H}$ and adhesion of 2 on the surface of inorganic glass is obtained.
\end{abstract}

\section{Introduction}

Transparent and hard films on various substrates have received considerable attention for optical applications. Inorganic coating films have excellent hardness, abrasion resistance and thermal resistance, but in addition to high cost, they are brittle and need high processing temperature [1]. Though some organic materials have been used to prepare the hard coating films, they have exhibited a relatively poor hardness [2]. In order to solve this problem, organic-inorganic hybrid materials have been introduced to prepare this transparent and hard coating film [3].

Indeed the $\mathrm{Al}-\mathrm{OH}$ on the surface of boehmite can occur condensation reaction with $\mathrm{Si}-\mathrm{OH}$ obtained by the hydrolysis of organosiloxane under a certain contain, and form Al-O-Si. Chemical bonds between boehmite and organosiloxane is achieved by this reaction, by which not only hybrid film combining the hardness of boehmite with the flexibility of organosiloxane can be obtained, but also the transparency of film is realized [2 4]. Therefore, over the past few years, there has been a growing interest in organosiloxane-boehmite hybrid coating film. However, the introduction of this material on an industrial scale is still in the stage of development for the high prices of boehmite.

To reduce cost and make the price more competitive, this paper attempts to replace boehmite with cheap aluminium isopropoxide (AIP), i.e., prepare hybrid film by AIP and organosiloxane directly, then modify this film with hydrolysis production of tetraethyl orthosilicate.

\section{Experiments}

\section{Materials}

$\gamma$-(2, 3-epoxypropoxyl) propyltrimethoxyl silane (GPTMS, chemically pure) was supplied by Guangzhou Baoxin Chemical Materials Co., Ltd; AIP (chemically pure) was supplied by Shanghai Fortune Bio-Technology Co., Ltd; Tetraethyl orthosilicate (TEOS, analytical reagent) was supplied by Tianjin Yongda Reagent Development Center.

\section{Preparation of Hydrolysis Productions}

Hydrolyzed GPTMS and TEOS solutions were prepared in the previous work respectively [5, 6].

\section{Preparation of GPTMS-boehmite Prepolymer}

A certain mass ratio of AIP, hydrolysis production of GPTMS and deionized water were put in a reactor equipped with a mechanical stirrer and reflux condenser. The mixture was heated to a certain temperature for a few hours with vigorous stirring to obtain GPTMS-boehmite prepolymer.

\section{Preparation of GPTMS-boehmite Hybrid Film}

The above prepolymer was coated on the surface of a $0.3 \mathrm{~cm} \times 9.0 \mathrm{~cm} \times 12.0 \mathrm{~cm}$ inorganic glass. 
After air drying $30 \mathrm{~min}$, the coated glass was placed in the oven with air circulation. Transparent film was prepared after curing $2.5 \mathrm{~h}$ at $125^{\circ} \mathrm{C}$.

\section{Modification of Hybrid Film with TEOS}

A certain mass ratio of GPTMS-boehmite prepolymer and hydrolyzed TEOS were put in a reactor, and then stirred stably under room temperature for $30 \mathrm{~min}$. Just like the preparation of GPTMS-boehmite hybrid film, the mixture was coated on the surface of an inorganic glass. After air drying $30 \mathrm{~min}$, the coated glass was placed in the oven with air circulation. Transparent film was prepared after curing $2 \mathrm{~h}$ at $110{ }^{\circ} \mathrm{C}$.

\section{Measurements}

The chemical bonds of coating film were evaluated by a Nexus470 Fourier transform infrared spectroscopy (FTIR) produced by America Thermo Nicolet Company. Pencil hardness and adhesion of coating film were carried out by GB/T5739-1996 and GB/T9286-1998 respectively.

\section{Results and Discussions}

\section{Influence of AIP Content}

When hydrolyzed GPTMS solution and deionized water were 100 and $75 \mathrm{~g}$ respectively, and the prepolymer was prepared at $80{ }^{\circ} \mathrm{C}$ for $13 \mathrm{~h}$, the influence of AIP content to properties of GPTMS-boehmite coating films is shown in Table 1. As can be seen, pencil hardness of coating film increases with the increase of AIP content while adhesion is almost the same. But it is found by the experiment that,when the content of AIP is higher than $12.5 \mathrm{~g}$, delamination is occurred in prepolymer, and transparent coating film can't be achieved accordingly. Therefore, $12.5 \mathrm{~g}$ was determined as the content of AIP in following work.

Tab. 1 Influence of AIP content to properties of coating films

\begin{tabular}{ccccc}
\hline AIP /g & 0 & 6.5 & 9.5 & 12.5 \\
\hline Pencil hardness & $3 \mathrm{~B}$ & $\mathrm{~B}$ & $\mathrm{HB}$ & $\mathrm{F}$ \\
Adhesion & 1 & 3 & 3 & 3 \\
\hline
\end{tabular}

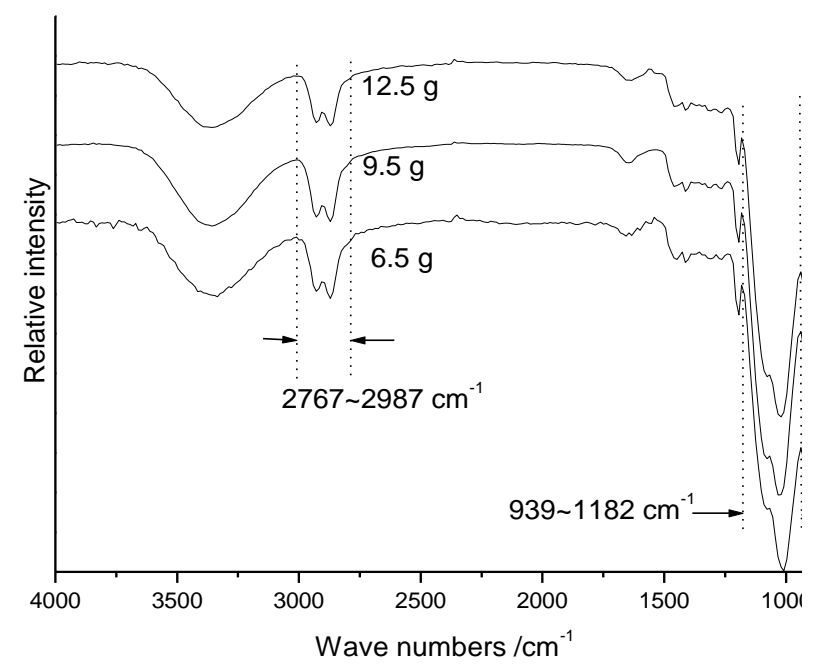

Fig.1 FTIR spectra of hybrid films with different AIP content

Figure 1 is FTIR spectra of GPTMS-boehmite coating films with different AIP content. Regarding stretching vibration peak of $\mathrm{C}-\mathrm{H}$ bond as internal standard, the influence of AIP content to the contents of Si-O-Si and Si-O-Al was researched (see Table 2). Here $S_{\mathrm{m} 1}$ is the integral area of 
Si-O-Si and Si-O-Al peaks of the film that $m \mathrm{~g}$ AIP is added. The integral range is $939 \sim 1182 \mathrm{~cm}^{-1}$. And $S_{\mathrm{m} 2}$ is the integral area of stretching vibration peak of C-H bond of the film that $m \mathrm{~g}$ AIP is added. The integral range is $2767 \sim 2987 \mathrm{~cm}^{-1} . S_{\mathrm{m}}$ is the ratio of $S_{\mathrm{m} 1}$ to $S_{\mathrm{m} 2}$, which represents the ratio of peaks area of $\mathrm{Si}-\mathrm{O}-\mathrm{Si}$ and $\mathrm{Si}-\mathrm{O}-\mathrm{Al}$ to that of $\mathrm{C}-\mathrm{H}$ bond. It can be seen from Table 2 that, the variation of $S_{\mathrm{m}}$ with AIP content is coincident with that of the hardness (see Table 1), which shows that the variation of hardness is controlled by the contents of Si-O-Si and Si-O-Al.

Tab. 2 Transformation of Si-O-Si and Si-O-Al by FTIR spectral analysis with AIP content

\begin{tabular}{cccc}
\hline $\mathrm{AIP} / \mathrm{g}$ & $S_{\mathrm{m} 1}$ & $S_{\mathrm{m} 2}$ & $S_{\mathrm{m}}$ \\
\hline 6.5 & 3423.75 & 827.41 & 4.14 \\
\hline 9.5 & 3997.26 & 820.79 & 4.87 \\
\hline 12.5 & 3767.60 & 672.29 & 5.60 \\
\hline
\end{tabular}

\section{Influence of Deionized Water Content}

When hydrolyzed GPTMS solution is $100 \mathrm{~g}$, and the prepolymer was prepared at $80{ }^{\circ} \mathrm{C}$ for $13 \mathrm{~h}$, the influence of deionized water content to properties of GPTMS-boehmite coating films is shown in Table 3. As can be seen, both pencil hardness and adhesion of coating film reduce with the increase of water content. But experiment shows that when water content is lower than $75 \mathrm{~g}$, hydrolysis effect of AIP becomes worse, and prepolymer appears delamination. Finally $75 \mathrm{~g}$ is determined as the content of water in following work.

Tab. 3 Influence of deionized water content to properties of coating films

\begin{tabular}{ccccc}
\hline $\mathrm{H}_{2} \mathrm{O} / \mathrm{g}$ & 50 & 75 & 100 & 125 \\
\hline Pencil hardness & $\mathrm{H}$ & $\mathrm{F}$ & $\mathrm{HB}$ & $\mathrm{B}$ \\
\hline Adhesion & 3 & 3 & 4 & 4 \\
\hline
\end{tabular}

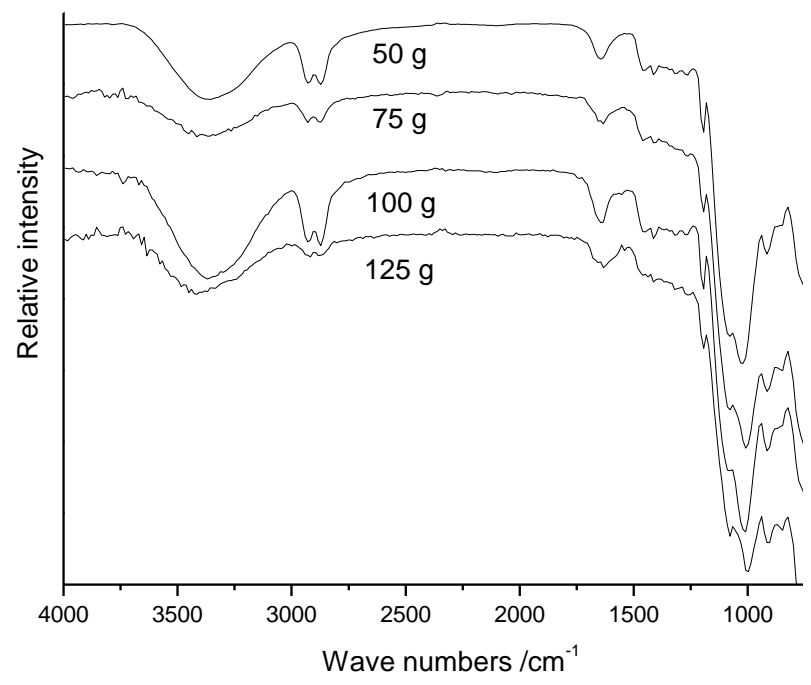

Fig. 2 FTIR spectra of films with different water content

FTIR spectra of GPTMS-boehmite coating films with different deionized water content are shown in Figure 2. Just like Figure 1, it was worked out also from Figure 2 that the variation of hardness of film is controlled by the contents of Si-O-Si and Si-O-Al. Here will not elaborate.

\section{Influence of Reaction Temperature}

When the prepolymer was prepared for $13 \mathrm{~h}$, The influence of preparation temperature to 
properties of GPTMS- boehmite coating films is shown in Table 4. As can be seen, hardness of film increases with the increase of preparation temperature while adhesion increases first, then declines. It was found by the experiment that when the temperature is lower $\left(75^{\circ} \mathrm{C}\right)$, the reaction effect of hydrolyzed GPTMS and AIP becomes worse, and prepolymer appears delamination. When the temperature is higher $\left(85{ }^{\circ} \mathrm{C}\right)$, viscosity of prepolymer becomes high, and prepolymer is e asy to form gel, which leads to a more difficult of process control. Hence, reaction temperature in following work was determined to be $80{ }^{\circ} \mathrm{C}$ finally.

Tab. 4 Influence of preparation temperature to properties of coating films

\begin{tabular}{cccc}
\hline Preparation temperature $/{ }^{\circ} \mathrm{C}$ & 75 & 80 & 85 \\
\hline Pencil hardness & $\mathrm{B}$ & $\mathrm{F}$ & $\mathrm{H}$ \\
\hline Adhesion & 4 & 3 & 5 \\
\hline
\end{tabular}

\section{Influence of Reaction Time}

Table 5 is the influence of reaction time to properties of GPTMS- boehmite coating films. As can be seen, pencil hardness of film increases with the extending of reaction time while adhesion increases first, then declines. Experiment also shows that, just like the influence of reaction temperature, when reaction time is too short, interaction of hydrolyzed GPTMS and AIP is poor, and prepolymer is easy to occur delamination. When reaction time is too long, it is easy to appear gel. Thus reaction time was determined to be $13 \mathrm{~h}$ according to the properties of film.

Tab. 5 Influence of reaction time to properties of coating films

\begin{tabular}{cccccc}
\hline Reaction time $/ \mathrm{h}$ & 7 & 9 & 11 & 13 & 15 \\
\hline Pencil hardness & $2 \mathrm{~B}$ & $\mathrm{~B}$ & $\mathrm{HB}$ & $\mathrm{F}$ & $\mathrm{H}$ \\
\hline Adhesion & 5 & 4 & 3 & 3 & 4 \\
\hline
\end{tabular}

According to the above study, the process that preparing GPTMS-boehmite prepolymer is obtained: AIP, hydrolyzed GPTMS solution and deionized water (the mass ratio were $12.5: 100: 75$ ) are put together and churned up $13 \mathrm{~h}$ under $80{ }^{\circ} \mathrm{C}$.

\section{Influence of Hydrolyzed TEOS Content}

Table 6 is the influence of hydrolysis TEOS content to properties of GPTMS-boehmite coating films. As can be seen, both pencil hardness and adhesion of film increase obviously with the increase of hydrolyzed TEOS, which is consistent with what we expected. But experiment shows that, hydrolysis product of TEOS itself is very brittle after drying, film can't form when the content of hydrolyzed TEOS is higher than $20 \%$ by weight. Therefore, the content of hydrolyzed TEOS was determined to be $20 \%$, correspondingly pencil hardness and adhesion of film reach $4 \mathrm{H}$ and 2 respectively, which means that coating film prepared here has the same properties as that prepared by GPTMS and boehmite while cost is reduced [7].

Tab. 6 Influence of TEOS content to properties of coating films

\begin{tabular}{cccccc}
\hline Hydrolyzed TEOS/prepolymer & 0 & 0.09 & 0.13 & 0.18 & 0.20 \\
\hline Pencil hardness & $3 \mathrm{~B}$ & $\mathrm{H}$ & $2 \mathrm{H}$ & $3 \mathrm{H}$ & $4 \mathrm{H}$ \\
Adhesion & 5 & 4 & 3 & 2 & 2 \\
\hline
\end{tabular}

\section{Conclusion}

Based on the research of the process, cheap GPTMS-boehmite transparent and hard coating film modified by hydrolyzed TEOS was prepared. AIP, hydrolyzed GPTMS and deionized water (the mass ratio were $12.5: 100: 75)$ are put together and vigorous stirred $13 \mathrm{~h}$ under $80{ }^{\circ} \mathrm{C}$. Then add 
$20 \%$ hydrolyzed TEOS and stir stably under room temperature for $30 \mathrm{~min}$. Curing the above mixture $2 \mathrm{~h}$ at $110{ }^{\circ} \mathrm{C}$ on the surface of inorganic glass, transparent coating film with a pencil hardness of $4 \mathrm{H}$ and adhesion of 2 can be obtained.

\section{Acknowledgment}

The paper was supported by No. 11164012 of the National Natural Science Foundation of China, No. 2012-2-105 of Science and Technology Project of Lanzhou Science and Technology of Bureau, and No. 1ZCU-XZ2014-14 Scientific Research Foundation of Lanzhou City University.

\section{References}

[1] S. J. Jeon, J. J. Lee, W. Kim, S. C. Tae, M. K. Sang. Hard coating films based on organosilane-modified boehmite nanoparticles under UV/thermal dual curing, Thin Solid Films. 516 (2008) 3904-3909.

[2] S. Sepeur, N. Kunze, B. Werner, H. Schmidt. UV curable hard coatings on plastics, Thin Solid Films. 351 (1999) 216-219.

[3] K. Chen, B. G. Li, G. M. Zeng. Development of the polymer/boemite nano-composites, Engineering Plastics Application. 37 (2009) 82-84.

[4] D. U. Wienhol, U Westerwelle. New routes to sol-gel systems: Polymer-based process extends scope of organic-inorganic coatings, European coatings journal. 7-8 (2006) 40-45.

[5] K. Chen, T. Y. Zhang, X. G. Cao, Y. Yang. Hydrolysis study on organic silicon oxygen alkyl GPTMS, New Chemical Materials. 39 (2011) 96-98.

[6] K. Chen, T. Y. Zhang, Y. J. Zhao, Y. Yang. Hydrolysis with TEOS, Joural of Lanzhou University of Technology. 37 (2011) 74-76.

[7] K. Chen, T. Y. Zhang, X. G. Cao, Y. Yang. The preparation of organoalkoxysilane/boehmite film, Paint \& Coatings Industry. 41 (2011) 9-13. 